Pacific Journal of Mathematics

CONVERGENCE THEOREMS FOR SOME SCALAR VALUED
INTEGRALS WHEN THE MEASURE IS NEMYTSKII 


\title{
CONVERGENCE THEOREMS FOR SOME SCALAR VALUED INTEGRALS WHEN THE MEASURE IS NEMYTSKII
}

\author{
A. De Korvin and C. E. Roberts JR.
}

One of the main potential applications of Hammerstein operators is a functional analytic study of nonlinear differential equations. In fact, some connections have already been established with equations of the form $\dot{x}(t)=\phi[x(t)]$ or $\dot{x}(t)=\phi[x(t), t]$. Other applications have been made to generalized random processes and the theory of fading memory in continuum mechanics. The main purpose of the present paper is to establish and study the representation of Hammerstein operators on continuous functions. A "nonlinear" integral is introduced for this purpose. Convergence theorems for a.e. and convergence in measure are established and contrasted. The last result of the paper relates uniform integrability, a key concept in the study of martingales, to essential ranges, an important concept used to establish the differentiability of some set functions.

1. Introduction. One of the main potential applications of Hammerstein operators, as stated in [12], is a functional analytic study of nonlinear differential equations. In fact the properties of the function $\phi$ required to insure the existence of solutions to the differential equations

$$
\dot{x}(t)=\phi[x(t)] \text { or } \dot{x}(t)=\phi[x(t), t]
$$

are closely related to the properties of the kernel $\phi$ used to represent an abstract Hammerstein operator $T$ as in [12]. The representation there is given by the formula

$$
T(f)=\int \phi[f(t), t] d \mu(t) .
$$

Other applications have already been initiated, we mention two of these; applications to the theory of generalized random processes in [9] and to the theory of fading memory in continuum mechanics in [5].

The above considerations motivated other work on the representation of Hammerstein operators over different function spaces. The reader is referred to [1], [2] and [3] for the cases where the function space is $L^{1}, M_{E}(\mathscr{B})$ or $C(K, X)$. The representation obtained in [1], [2] and [3] is of the form 


$$
T(f)=\int f d m
$$

where the integral is (necessarily) nonlinear. Here $m$ denotes a finitely additive set function defined on an appropriate ring with values in $M(X, C)$ which is a certain space of maps (not necessarily linear) from $X$ (a Banach space) into $C$ (the scalar field). The integral is of course suitably defined. These representations should be contrasted with the representation obtained in [12] where a kernel function is present and where the integral is the standard one. The nonlinearity imposed on the operator $T$ in all of the above works is:

$$
T\left(f+f_{1}+f_{2}\right)=T\left(f+f_{1}\right)+T\left(f+f_{2}\right)-T(f)
$$

whenever $f_{1}$ and $f_{2}$ have disjoint supports. This condition has been called the Hammerstein property by J. Batt in [3] and the additive property by N. A. Friedman and A. E. Tong in [8].

The main purpose of the present paper is to study the properties of the nonlinear integral as defined in [1], [2] and [3]. The main interest here focuses on Hammerstein operators that are scalar valued and defined over $C(K, X)$, the space of continuous functions over the compact space $K$ under the supremum norm and with values in the Banach space $X$. Thus in our study of the properties of $\int f d m, f$ will be defined over $K$ with values in $X$ and $m$ will be a finitely additive set function with values in $M(X, C)$ and will satisfy certain continuity conditions. Actually most of our results can immediately be generalized to abstract Nemytskii measures (see [2]) since continuity of the functions will not be used in most of the proofs. It should be recalled that if $T$ is a Hammerstein operator on $C(K, X)$, then $T(f)=\int f d m_{T}$ where $m_{T}$ has values in $M(X, C)$ and moreover $m_{T}$ is a measure extendable to $\mathscr{B}$, the Borel field of $K$ (see [3]). Two types of convergence will be studied here. The first convergence will be $s_{n} \rightarrow f m$ a.e. where $\left\{s_{n}\right\}$ is a sequence of simple $X$-valued functions. The key property in this type of convergence will be the requirement that $\int_{(\cdot)} s_{n} d m$ be uniformly countably additive measures. An important technical device introduced will be a version of the Egoroff Theorem. The last theorem obtained for $m$ a.e. convergence states that if $T$ is a scalar valued Hammerstein operator on $C(K, X)$ that has a $G$-representation (see R. K. Goodrich [10]) with respect to $m^{\prime}$, then $m^{\prime}(\cdot) x$ is necessarily uniformly countably additive for $\|x\| \leqq \alpha$ provided the bounded or the dominated convergence theorem holds for $m^{\prime}$. The second type of convergence to be studied is convergence in 
measure. It is appropriate here to recall that W. V. Smith and D. H. Tucker [15] and also D. H. Tucker and S. G. Wayment [16] have constructed examples of $X$-valued functions converging in measure but having no subsequence converging $m$ a.e. (and even an example of a sequence that converges to different functions in measure and $m$ a.e.!) For convergence in measure we use the concept of essential range introduced by M. A. Rieffel [13] to obtain the Radon-Nikodym Theorem for Bochner integrals. Another central concept is uniform integrability. Uniform integrability is known to play a key role for martingale convergence theorems, for example see [4]. The last theorem obtained in the present paper states that if $f_{n}$ are essential range functions and $\int_{A} f_{n} d m$ converges to 0 uniformly for $A \in \mathscr{B}$ then the $f_{n}$ converge to 0 in measure provided the essential ranges of $f_{n}$ are bounded away from 0 over appropriate sets and provided they transfer uniform integrability. Of course it is easy to give an example where $\int_{A} f_{n} d m$ converges to 0 but $f_{n}$ does not converge to 0 in measure, even if $m$ is linear. For example let $m(A)(x, y)=(\lambda(A) x, 0)$ where $(x, y) \in R^{2}$ and where $\lambda$ is the Lebesgue measure and let $f_{n}(t)=(0, n)$. Then $\int_{A} f_{n} d m=0$ yet $f_{n}$ does not converge to 0 in measure. (See W. V. Smith [14], for example.)

We now present a summary of the results obtained. All of these results pertain to measures representing Hammerstein operators $T$. To stress this we will use the notation $m_{T}$ in the section on results. The first result states that if $\left\{s_{n}\right\}$ is a sequence of $X$ valued simple functions converging $m$ a.e. to $f$ and if the measures $\int_{(\cdot)} s_{n} d m$ are uniformly countably additive, then there exists a unique scalar measure $r$ such that $r(E)=\lim \int_{E} s_{n} d m$, moreover the limit is uniform for $E \in \mathscr{B}$. This allows us to define the space $L^{1}(m)$ as the space of functions from $K$ into $X$ that are limits $m$ a.e. of a sequence of simple functions $s_{n}$ with $\int_{(\cdot)} s_{n} d m$ uniformly countably additive. We then show that $\left\{s_{n}\right\}$ may be replaced by $\left\{f_{n}\right\}$ where $f_{n} \in L^{1}(m)$. The second result is an Egoroff type theorem. The norm used is of the type $\sup _{\|x\| !}\left|s_{n}(\cdot) x-f(\cdot) x\right|$ where $s_{n}$ and $f$ are scalar valued and $x$ is in $X$. As a corollary to this result we show that if in addition $\left\{\int_{(\cdot)} s_{n}(\cdot) x d m\right\}$ is uniformly countably additive (in $n$ and for $\|x\| \leqq \alpha)$, then there exists a scalar measure $r_{x}$ such that $\sup _{\|x\| \leq \alpha}\left|r_{x}(E)-\int_{E} s_{n}(\cdot) x d m\right|$ converges to 0 uniformly for $E \in \mathscr{B}$. The third result shows a version of the bounded convergence theorem for the nonlinear integral. This result is followed by a version of the dominated convergence theorem. Our next result 
relates to $G$-representation as defined by R. K. Goodrich, see [10]. For our second type of convergence we use the $\alpha$-semi-variation function to define convergence in measure. We already have discussed the examples obtained in [15] and [16] to point out the rather striking differences between the two modes of convergence. We define the function $f$ to be $m$-integrable if there exists a sequence $\left\{s_{n}\right\}$ of simple functions that are uniformly integrable (see [4]) and such that $\left\{s_{n}\right\}$ converges to $f$ in measure. It is then shown that $\int_{A} s_{n} d m$ converges uniformly for $A \in \mathscr{B}$. Our last result, already mentioned above, used the essential ranges of $\left\{f_{n}\right\}$ (see [13]) and the property of uniform integrability to yield a sufficient condition for the sequence $\left\{f_{n}\right\}$ to converge to 0 in measure.

II. Results. We introduce some basic notations. Let $K$ denote a compact set, $\mathscr{B}$ the Borel sets of $K, C(K, X) X$-valued functions that are continuous and defined on $K$ with the topology of the sup norm. Here $X$ denotes a Banach space. Let $u$ be a function from $X$ into $C$. For $\alpha>0$ let $u_{\alpha}$ denote the restriction of $u$ to the closed $\alpha$-ball of $X$. Let $\left\|u_{\alpha}\right\|=\sup \|u(x)\|$ where the sup is over $\|x\| \leqq \alpha$. For $\delta>0$ define

$$
D_{\delta} u_{\alpha}=\sup \|u(x)-u(y)\| \text { where the sup is over }
$$

$\|x\| \leqq \alpha,\|y\| \leqq \alpha,\|x-y\| \leqq \delta$. Let $M(X, C)$ denote the space of all functions from $X$ into $C$ which are bounded on the $\alpha$-balls of $X$, uniformly continuous on bounded sets of $X$ and 0 at 0 . Thus if $u \in M(X, C)$ then

$$
u(0)=0,\left\|u_{\alpha}\right\|<\infty, \lim _{\delta \rightarrow 0} D_{\hat{\delta}} u_{\alpha}=0 .
$$

Let $m$ be a finitely additive function from $\mathscr{B}$ into $M(X, C)$. We set $m_{\alpha}(B)=m(B)_{\alpha}$ and define

$$
s v\left[m_{\alpha}, B\right]=\sup \left\|\Sigma m\left(B_{i}\right) x_{i}\right\| .
$$

Here the sup is over finite partitions $\left\{B_{i}\right\}$ of $B$ with $\left\|x_{i}\right\| \leqq \alpha$. Let

$$
s v_{\hat{o}}\left(m_{\alpha}, B\right)=\sup \left\|\Sigma m\left(B_{i}\right) x_{i}-\Sigma m\left(B_{i}\right) y_{i}\right\|
$$

where the sup is over finite partitions $\left\{B_{i}\right\}$ of $B$ and $\left\|x_{i}\right\| \leqq \alpha$, $\left\|y_{i}\right\| \leqq \alpha,\left\|x_{i}-y_{i}\right\| \leqq \delta$. Unless otherwise stated $m$ denotes a finitely additive set function from $\mathscr{B}$ into $M(X, C)$ satisfying

$$
s v\left[m_{\alpha}, K\right]<\infty \text { and } \lim _{\delta \rightarrow 0} s v_{\delta}\left[m_{\alpha}, K\right]=0 .
$$

A property will be called true $m$ a.e. if the property is true for all $t \notin A$ and if whenever $B \subset A$ and $B \in \mathscr{B}$ then $m(B)=0$. Let 
$\chi_{C_{j}}$ represents the characteristic function of $C_{i}$. For disjoint $C_{\imath}$ we define

$$
\int \Sigma \chi_{C_{i}} x_{i} d m=\Sigma m\left(C_{i}\right) x_{i}
$$

$M_{I I}[C(K, X), C]$ will denote all scalar valued maps defined on $C(K, X)$ and satisfying

$$
T\left(f+f_{1}+f_{2}\right)=T\left(f+f_{1}\right)+T\left(f+f_{2}\right)-T(f)
$$

whenever $f_{1}$ and $f_{2}$ have disjoint supports

$$
\begin{gathered}
T(0)=0 \\
\left\|T_{\alpha}\right\|<\infty \\
\lim _{\hat{o} \rightarrow 0} D_{\hat{o}} T_{\alpha}=0 \text { where } D_{\hat{o}} T_{\alpha}=\sup \left\|T x_{i}-T y_{i}\right\| \text { and }
\end{gathered}
$$

where the sup is over $\|x\| \leqq \alpha,\|y\| \leqq \alpha,\|x-y\| \leqq \delta$. In [3], J. Batt has shown that if $T \in M_{H}[C(K, X), C]$, then $T$ can be written as $T(f)=\int f d m$ where the nonlinear integral is extended from simple functions to functions in $C(K, X)$. Moreover $\left\|T_{\alpha}\right\|=s v\left[m_{\alpha}, K\right]$ and $D_{\hat{o}} T_{\alpha}=s v_{\hat{o}}\left[m_{\alpha}, K\right]$. If $T \in M_{I I}[C(K, X), C], m_{T}$ will denote the corresponding measure. We now prove our first result.

Theorem 1. Let $\left\{s_{n}\right\}$ be a sequence of $X$-valued simple functions. Assume $\left\{s_{n}\right\}$ converges to $f$ pointwise and assume that the set functions $\int_{(\cdot)} s_{n} d m_{T}$ are uniformly countably additive. Then there exists a unique, countably additive, scalar measure $r$ such that $r(E)=\lim _{n \rightarrow \infty} \int_{E} s_{n} d m_{T}$ uniformly for $E \in \mathscr{S}$.

Proof. For every $E \in \mathscr{\mathscr { S }}$ we define

$$
\hat{m}(E)=\sum_{n=1}^{\infty} \frac{\int_{E} s_{n} d m_{T}}{2^{n}\left[1+\sup _{A \in \mathscr{D}}\left|\int_{A} s_{n} d m_{T}\right|\right]}
$$

clearly $\hat{m}$ is a finitely additive set function on $\mathscr{B}$. It is shown in [3] that $m_{T}(\cdot) x$ is countably additive for $x \in X$. In fact it is uniformly countably additive for $\|x\| \leqq \alpha$. It follows that $\hat{m}$ is a bounded countably additive function on $\mathscr{B}$. Since $\left\{s_{n}\right\}$ converges to $f$, by Corollary 1 to Egoroff's Theorem (see [6, p. 95]). there exists a sequence $\left\{A_{k}\right\}$ of disjoint sets of $\mathscr{B}$ and $N \in \mathscr{B}$ such that $\left\{s_{n}\right\}$ converges uniformly to $f$ on each $A_{k}, N$ is a $\hat{m}$-null set, and $K=$ $N \cup \cup A_{k}$. Thus, $\int_{N} s_{n} d m_{T}=0$ for all $n$. Let $B_{k}=\bigcup_{j=1}^{k} A_{j}$, clearly $B_{k} \uparrow N^{\prime}$ and the convergence is uniform on each $B_{k}$. Moreover, 


$$
\begin{aligned}
\left|\int_{E}\left(s_{n}-s_{p}\right) d m_{T}\right| & \leqq\left|\int_{E \cap B_{k}}\left(s_{n}-s_{p}\right) d m_{T}\right|+\left|\int_{E \cap B_{k}^{\prime} \cap N^{\prime}}\left(s_{n}-s_{p}\right) d m_{T}\right| \\
& \leqq s v_{\tau_{k}}\left[m_{T \alpha}, K\right]+\left|\int_{E \cap B_{k}^{\prime} \cap N^{\prime}} s_{n} d m_{T}\right|+\left|\int_{E \cap B_{k}^{\prime} \cap N^{\prime}} s_{p} d m_{T}\right|
\end{aligned}
$$

where $\tau_{k}=\operatorname{ess} \sup \left\|s_{n}-s_{p}\right\|$ restricted to $B_{k}$ and where $\alpha=\sup \left|x_{i}\right|$ where $x_{i}$ is a value in Range $s_{n} \cup$ Range $s_{p}$ where $s_{n}$ and $s_{p}$ are restricted to $E \cap B_{k}$. The first term on the right goes to 0 as $n$ and $p$ get large. The next two terms tend to 0 by uniform countable additivity of $\int_{(\cdot)} s_{n} d m_{T}$. Thus $\int_{E} s_{n} d m_{T}$ converges uniformly for $E \in \mathscr{B}$ and

$$
r(E)=\lim _{n \rightarrow \infty} \int_{E} s_{n} d m_{T}
$$

has the stated properties.

Note. The above proof can be extended to the case in which pointwise convergence of $\left\{s_{n}\right\}$ is replaced by $m_{T}$ a.e. convergence.

We now define $\int_{E} f d m_{T}=\lim \int_{E} s_{n} d m_{T}$. The arguments above show that $\int_{E} f d m_{T}$ is well defined. Let $L^{1}\left(m_{T}\right)$ denote all functions $f: K \rightarrow X$ where $f$ is the limit of a sequence $\left\{s_{n}\right\}$ of simple functions $m_{T}$ a.e. and where $\int_{(\cdot)} s_{n} d m_{T}$ are uniformly countably additive.

Proposition 1. If $f_{n} \in L^{1}\left(m_{T}\right)$ and $\left\{f_{n}\right\}$ converges to $f m_{T}$ a.e. and $\int_{(\cdot)} f_{n} d m_{T}$ are uniformly countably additive, then $f \in L^{1}\left(m_{T}\right)$ and $\int_{E} f_{n} d m_{T}$ converges to $\int_{E} f d m_{T}$ uniformly for $E \in \mathscr{B}$. Moreover

$$
\left|\int_{E} f d m_{T}\right| \leqq s v_{\|f\| E}\left[m_{T}, B\right] \text { where }\|f\|_{E} \text { is }
$$

the essential sup of $f$ restricted to $E$.

Proof. The first part of the proof follows from the fact that the proof of Theorem 1 remains true when $\left\{f_{n}\right\}$ replaces $\left\{s_{n}\right\}$. In addition it is clear that a sequence of simple functions $\left\{s_{n}\right\}$ may be found which converges to $f$ and for which $\left\{\int_{(.)} s_{n} d m_{T}\right\}$ is uniformly countably additive. Now:

$$
\begin{aligned}
\left|\int_{E} f d m_{T}\right| & \leqq\left|\int_{E}\left(f-s_{n}\right) d m_{T}\right|+\left|\int_{E} s_{n} d m_{T}\right| \\
& \leqq\left|\int_{E}\left(f-s_{n}\right) d m_{T}\right|+\left|\int_{B_{k} \cap E} s_{n} d m_{T}\right|+\left|\int_{E \cap B_{k}^{\prime} \cap N^{\prime}} s_{n} d m_{T}\right|
\end{aligned}
$$




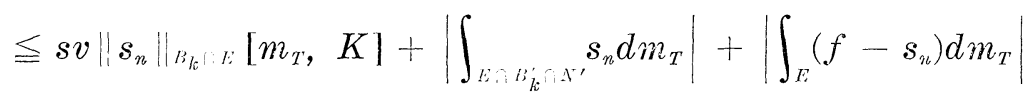

where $B_{i}$ are as in Theorem 1. As $n \rightarrow \infty$, the first term goes to $s v\|f\|_{B, i n}$ and the last term converges to 0 . As $k \rightarrow \infty$ the middle term converges uniformly to 0 in $n$ and the first term converges to $s v\|f\|_{E}$.

We now prove a version of the Egoroff Theorem which is suitable for our purpose.

THEOREM 2 (Egoroff). Let $\left\{s_{n}\right\}$ be a sequence of real valued simple functions, let $f$ be a real valued function and assume that

$$
\sup _{|x| 1 \leq \alpha}\left|s_{n}(\cdot) x-f(\cdot) x\right| \text { converges to } 0 m_{T} \text { a.e. }
$$

Then there exists a sequence $\left\{A_{k}\right\}$ of disjoint sets of $\mathscr{B}$ and $N \in \mathscr{B}$ such that

(1) $K=N \cup \cup_{k} A_{k}$.

(2) $\sup _{\|x\|_{1} \mid}\left|s_{n}(\cdot) x-f(\cdot) x\right|$ converges to 0 uniformly on $A_{k}$.

(3) $\int_{\mathrm{V}} s_{n}(\cdot) x d m=0$ for all $n$ and $\|x\| \leqq \alpha$.

Proof. Let

$$
\hat{m}_{x}(E)=\sum_{n}\left[\frac{\int_{E} s_{n}(\cdot) x d m_{T}}{1+\sup _{A \in \mathscr{S}}\left|\int_{A} s_{n}(\cdot) x d m_{T}\right| 2^{n}}\right] \text { For each fixed } x \text { with }
$$

$\|x\| \leqq \alpha, \hat{m}_{*}$ is finitely additive, bounded and since $m_{\alpha}(\cdot)$ is countably additive, it follows that $\hat{m}_{x}(\cdot)$ is uniformly countably additive for $\|x\| \leqq \alpha$. Since $\hat{m}_{x}$ is uniformly bounded it follows that $\left\{m_{x} \mid\|x\| \leqq \alpha\right\}$ is weakly sequentially compact (see [7, p. 305]). Thus there exists a positive measure $\lambda_{\alpha}$ where $\lim _{\lambda_{\alpha}(E) \rightarrow 0} \hat{m}_{x}(E)=0$ uniformly for $\|x\| \leqq \alpha$. Also $\lambda_{\alpha}$ may be chosen so that $\lambda_{\alpha}(E) \leqq$ $\sup _{\|x\| \leq \alpha}\left|\hat{m}_{x}(E)\right|$ (see [7, p. 307].). Since $\sup _{\|x\| \leq_{\alpha}}\left|s_{n}(\cdot) x-f(\cdot) x\right|$ converges to $0 \hat{m}_{x}$ a.e. for $\|x\| \leqq \alpha$, it converges to $0, \lambda_{\alpha}$ a.e. The proof then proceeds as in Theorem 1.

Corollary 1. Let $\left\{s_{n}\right\}$ and $f$ be as above. Assume $\int_{(\cdot)} s_{n}(\cdot) x d m_{T}$ are uniformly countably additive with respect to $n$ and for $\|x\| \leqq \alpha$. Then there exists scalar measures $r_{x}$ such that

$$
\sup _{1, x \leq x}\left|r_{x}(E)-\int_{E} s_{n} d m_{T}\right| \text { converges to } 0 \text { uniformly }
$$


for $E \in \mathscr{B}$ and $\|x\| \leqq \alpha$.

Proof. Let $\left\{A_{k}\right\}$ and $N$ be as previously defined. Let

$$
\begin{aligned}
B_{k}= & \bigcup_{j=1}^{k} A_{j} \sup _{\mid\|x\| \leqq \alpha}\left|\int_{E}\left(s_{n}-s_{p}\right)(\cdot) x d m_{T}\right| \\
\leqq & \sup _{\|x\| \leqq \alpha}\left|\int_{E \cap B_{k}}\left(s_{n}-s_{p}\right)(\cdot) x d m_{T}\right|+\sup _{\|x\| \leqq \alpha}\left|\int_{E \cap B_{k}^{\prime} \cap N}\left(s_{n}-s_{p}\right)(\cdot) x d m_{T}\right| \\
\leqq & s v \beta_{(n, p)}\left[m_{T}, K\right]+\sup _{\|x\| \leqq \alpha}\left|\int_{E \cap B_{k}^{\prime} \cap N^{\prime}} s_{n}(\cdot) x d m_{T}\right| \\
& \quad+\sup _{|| x \mid ! \alpha}\left|\int_{E \cap B_{k}^{\prime} \cap N} s_{p}(\cdot) x d m_{T}\right|
\end{aligned}
$$

where $\beta(n, p)=\sup _{\|x\| \leqslant \alpha} \operatorname{ess} \sup \left\|s_{n}(\cdot) x \chi_{B_{k}}-s_{p}(\cdot) x \chi_{B_{k}}\right\|$. Hence $\lim _{n \rightarrow \infty} \rightarrow \infty(n, p)=0$, the last two terms converge to 0 by uniform countable additivity.

Note. We may write $\int_{E} f(\cdot) x d m_{T}=r_{x}(E)$.

Let $M(K)$ denote all scalar valued functions on $K$ that are limits $m_{T}$ a.e. of simple functions.

THEOREM 3. Let $\left\{f_{n}\right\}$ be a sequence in $M(K)$. Assume that $\left\{f_{n}(\cdot) x\right\}$ converges to $f(\cdot) x m_{T}$ a.e. uniformly for $\|x\| \leqq \alpha$. Assume also that $\left\{f_{n}\right\}$ is uniformly bounded by some constant $M$. Then

$$
\left\{\int_{E} f_{n}(\cdot) x d m_{T}\right\} \text { converges to } \int_{E} f(\cdot) x d m_{T}
$$

uniformly for $E \in \mathscr{B}$.

Proof. Without loss of generality assume $f_{n} \geqq 0$. (Note that $f_{n}^{+}$and $f_{n}^{-}$have disjoint supports.) Let $\left\{g_{n, i}\right\}$ be a sequence of simple functions such that $g_{n, i} \uparrow f_{n}$. Then

$$
g_{n, i}=\sum_{i=1}^{k_{n}} a_{i}^{n} \chi_{E_{i}(n)}
$$

and

$$
\int_{E} \boldsymbol{g}_{n, i} d m_{T}=\sum_{i=1}^{k_{n}} a_{i}^{n} m_{T}\left[E_{i}(n)\right] x .
$$

Let $\Sigma_{p}$ and $\Sigma_{N}$ denote sums over positive and negative terms.

$$
\begin{aligned}
\left|\sum_{i=1}^{k_{n}} a_{\imath}^{n} m_{T}\left[E_{i}(n)\right] x\right| & =\sum_{p} a_{i}^{n} m_{T}\left[E_{i}(n)\right] x-\sum_{N} a_{i}^{n} m_{T}\left[E_{i}(n)\right] x \\
& \leqq 2 M \operatorname{var} m_{T}(E) x
\end{aligned}
$$


Since $m_{T}(\cdot) x$ is countably additive (uniformly for $\|x\| \leqq \alpha$ ), the $\left\{\int_{(\cdot)} g_{n, i}(\cdot) x d m_{T}\right\}$ are uniformly countably additive (with respect to $n, i$ and for $\|x\| \leqq \alpha)$. Hence by Corollary $1, \lim _{i \rightarrow \infty} \int_{F} g_{n, i}(\cdot) x d m_{T}=$ $\int_{F} f_{n}(\cdot) x d m_{T}$ uniformly for $F \in \mathscr{B}$ and $\|x\| \leqq \alpha$. Also $\left\{\int_{(\cdot)} f_{n}(\cdot) x d m_{T}\right\}$ is uniformly countably additive. So again $\left\{\int_{E} f_{n}(\cdot) x d m_{T}\right\}$ converges to $\int_{E} f(\cdot) x d m_{T}$ uniformly in $E$.

Of course Theorem 3 is a version of the bounded convergence theorem for nonlinear integrals. We now obtain a version of the dominated convergence theorem.

Proposition 2. Let $\left\{f_{n}\right\}$ be a sequence in $L^{1}\left(m_{T}\right)$ and assume that $\left\{f_{n}\right\}$ converges to $f m_{r}$ a.e. Moreover assume

$$
\left|\int_{E} f_{n} d m_{T}\right| \leqq \sup _{F \subset E}\left|\int_{F} g d m_{T}\right| \text { where } g \in L^{1}\left(m_{T}\right) \text {. }
$$

Then $f \in L^{1}\left(m_{T}\right)$ and $\left\{\int_{E} f_{n} d m_{T}\right\}$ converges to $\int_{E} f d m_{T}$ uniformly for $E \in \mathscr{B}$.

Proof. Since $g \in L^{1}\left(m_{T}\right)$, by Theorem 1, $\int_{(\cdot)} g d m_{T}$ is countably additive and hence bounded. Since from the hypothesis $\int_{(\cdot)} f_{n} d m_{T}$ is dominated by the variation of $\int_{(\cdot)} g d m_{r}, \int_{(\cdot)} f_{n} d m_{T}$ are uniformly countably additive and by Proposition 1 the result follows.

We now proceed to establish a partial converse to the above result. In the work of $R$. K. Goodrich [10] the following ring of subsets plays a central role. Let $R$ be the ring of all subsets $E$ of $K$ for which there exist nonincreasing sequences of continuous functions $\left\{f_{n}\right\},\left\{g_{n}\right\}$ with $f_{n}-g_{n}$ converging to $\chi_{E}$. It is shown in [10] that $R$ is the ring generated by all compact $G_{\delta}$ subsets of $K$. Following [10], if $T$ is a (not necessarily linear) operator from $C(K, X)$ into scalars we say that $T$ has a $G$-type representation if

$$
T(f)=\int f d m^{\prime} \text { where } m^{\prime} \text { maps the ring } R \text { into } M(X, C)
$$

and where $m^{\prime}(\cdot) x$ is countably additive for each fixed $x$. (Of course, it is shown in [10] that every continuous linear operator on $C(K, X)$ has a $G$-type representation.)

We now consider two conditions related to the previous result.

(A) Theorem 3 is true with $m^{\prime}$ replacing $m_{T}$. 
(B) Let $\left\{f_{n}\right\}$ be a sequence of scalar valued functions converging $m^{\prime}$ a.e. to $f$ and assume that $\left|\int_{E} f_{n} d m^{\prime}\right| \leqq \sup _{F \subset E}\left|\int_{E} g d m^{\prime}\right|$ for some $g \in L^{1}\left(m^{\prime}\right)$. Then $\left\{\int_{E} f_{n}(\cdot) x d m^{\prime}\right\}$ converges to $\int_{E} f(\cdot) x d m^{\prime}$ for each fixed $x$.

Theorem 4. Let $T \in M_{H}[C(K, X), C]$. Assume that $T$ has a $G$-representation with respect to $\mathrm{m}^{\prime}$. Then under condition (A) or (B) $m^{\prime}(\cdot) x$ is uniformly countably additive for $\|x\| \leqq \alpha$.

Proof. Suppose (A) holds: Let $E_{i} \in R$ with $E_{i} \downarrow \varnothing$. Then $m^{\prime}\left(E_{i}\right) x$ converges to 0 for every fixed $x$. By the Kluvanek extension theorem [11], $m^{\prime}(\cdot) x$ has a unique countably additive extension to $\mathscr{B}$ and since by [3] $T$ admits the representation $T(f)=\int f d m$ where $m(\cdot) x$ is uniformly countably additive for $\|x\| \leqq \alpha$ and since $m(\cdot) x=m^{\prime}(\cdot) x$, it follows that $m^{\prime}$ has the same property. The same argument can be made under assumption of condition (B).

We now initiate a study of convergence in measure. In the introduction we mentioned the works of W. V. Smith and D. H. Tucker [15] and of D. H. Tucker and S. G. Wayment [16]. Their examples highlight the great difference between convergence a.e. and convergence in measure. We now define convergence in measure. Let $m_{T}$ be as above. We say $\left\{f_{n}\right\}$ converges to $f$ in measure if $s v_{\alpha} m_{T}\left\{\left\|f_{n}(\cdot)-f(\cdot)\right\| \geqq \delta\right\}$ converges to 0 as $n$ gets large, for every $\alpha>0$ and $\delta>0$ fixed. It is obvious, since the semi-variation is subadditive, that if $\left\{f_{n}\right\}$ converges to $f$ in measure then for every fixed $\varepsilon>0, \alpha>0$, and $\delta>0, s v_{\alpha} m_{T}\left\{\left\|f_{n_{1}}(\cdot)-f_{n_{2}}(\cdot)\right\| \geqq \delta\right\}<\varepsilon$ provided $n_{1} \geqq N$ and $n_{2} \geqq N$. Of course $N$ depends on $\varepsilon, \alpha, \delta$. We now define an $X$-valued function to be $m_{T}$-integrable if there exists a sequence $\left\{s_{n}\right\}$ of uniformly integrable simple functions such that $\left\{s_{n}\right\}$ converges to $f$ in measure and such that for every $\varepsilon>0, \alpha>0$, there exists a number $u$ (depending on $\varepsilon$ and $\alpha$ ) such that

$$
s v_{\alpha}\left[m_{T}, E\right]<u \text { implies }\left|\int_{E} s_{n} d m_{T}\right|<\varepsilon \text { i.e., }
$$

$\left\{\int_{(\cdot)} s_{n} d m_{T}\right\}$ is uniformly continuous with respect to the semi-variation. Recall that uniform integrability means that for every $\varepsilon>0$ there exists $K(\varepsilon)$ such that

$$
\left|\int_{\left\{\left\|s_{n}\right\|>K(\varepsilon)\right\} \cap A} s_{n} d m_{T}\right|<\varepsilon \text { for all } n .
$$

In the introduction we have already stated that uniform integrability has implications for the convergence of martingales. There 
are also strong implications for convergence properties in $L^{p}$. For example $\left\{g_{n}\right\}$ converges in $\mu$ measure to $g$ with $\left|g_{n}\right|^{p}$ uniformly integrable is equivalent to the norm convergence in $L^{p}$ (see $[4, \mathrm{p}$. 185] where conditions equivalent to uniform integrability are pointed out).

Proposition 3. Let $f$ be $m_{T}$ integrable. Let $s_{n}$ be as above. Then $\lim \int_{A} s_{n} d m_{T}$ exists uniformly for $A \in \mathscr{B}$.

Proof. Pick $\varepsilon>0$, let $K(\varepsilon)$ be chosen so that

$$
\left|\int_{\left\{11 s_{n}\{1 \geq K(\varepsilon)\}\right.} s_{n} d m_{T}\right|<\varepsilon / 4 \text { for all } n \text {. Now pick } u
$$

so that $s v_{K(\varepsilon)} m_{T}\left(E_{1}\right)<u$ implies $\left|\int_{E_{1}} s_{n} d m_{T}\right|<\varepsilon / 4$. Now pick $u^{\prime}$ so that

$$
s v_{u^{\prime}}\left[\left(m_{T}\right)_{K(\varepsilon)}, K\right]<\varepsilon / 4 .
$$

By the subadditivity of the semi-variation pick $N$ so that

$$
s v_{K(\varepsilon)} m_{T}\left\{\left\|s_{n_{1}}(\cdot)-s_{n_{2}}(\cdot)\right\|>u^{\prime}\right\}<u
$$

for $n_{1} \geqq N$ and $n_{2} \geqq N$.

Let $E_{2}=\left\{\left\|s_{n_{1}}(\cdot)-s_{n_{2}}(\cdot)\right\|>u^{\prime}\right\}$. Then

$$
\left|\int_{E_{2}} s_{n_{1}} d m_{T}-\int_{E_{2}} s_{n_{2}} d m_{T}\right|<\varepsilon / 2 \text { since }
$$

$s v_{K(\bullet)} m_{T}\left(E_{2}\right)<u$. Now

$$
\left|\int_{E_{2}^{\prime}} s_{n_{1}} d m_{T}-\int_{E_{2}^{\prime}} s_{n_{2}} d m_{T}\right| \leqq s v_{u^{\prime}}\left[m_{T}, K\right]+\varepsilon / 4+\varepsilon / 4<3 \varepsilon / 4
$$

Thus for all $A \in \mathscr{B}$,

$$
\left|\int_{A} s_{n_{1}} d m_{T}-\int_{A} s_{n_{2}} d m_{T}\right|<2 \varepsilon .
$$

Thus $\lim \int_{A} s_{n} d m_{T}$ exists uniformly as $A \in \mathscr{B}$.

Note. If we denote this limit by $m_{f}(A)$, then $m_{f}$ is a measure on $\mathscr{B}$ by the Nikodym theorem (see [7, p. 160]).

Let $f$ be a function from $K$ into $X$. By the essential range of $f$ over some set $E \subset K$ we mean $\left\{x \in X \mid m_{T}[\{\|x-f(\cdot)\|<\varepsilon\} \cap E] \neq 0\right\}$. We denote this set by $e r_{E}(f) . f$ will be called an essential range 
function if $e r_{F}(f) \cap f(F) \neq \phi$ for all $F \in \mathscr{B}$ that are not $m_{T}$-null sets. The set $\operatorname{er}_{E}(f)$ was introduced by M. A. Rieffel in [13]. It is shown there that if $f$ is the pointwise limit of a sequence of $X$ valued simple functions and if $\mu$ is a positive measure then $e r_{E}(f) \cap f(E) \neq \phi$ provided $\mu(E)>0$. The separability of $f(E)$ and the positivity of $\mu$ were both used in the proof of this result. $e r_{E}(f)$ is used in [13] as a bound on local average ranges which in turn play a key role in establishing the vector valued version of the Radon-Nikodym theorem.

Lemma. If for every pair $(A, B)$ of nonnull $m_{T}$ sets of $\mathscr{B}$

$$
e r_{A}(f) \cap f(A) \neq \phi \text { and } e r_{B}(g) \cap g(B) \neq \dot{\phi}
$$

where $f$ and $g$ are essential range functions and if $\|f(\cdot)-g(\cdot)\|<$ $a$ then for every $x \in e r_{E}(f)$ and $\varepsilon>0$ there exists $y \in e r_{E}(g)$ such that $\|y-x\|<a+\varepsilon$.

$$
\begin{aligned}
\text { Proof. } & m_{T}[\{x \in X \mid\|x-f(\cdot)\|<\varepsilon / 2\} \cap E] \neq \dot{\phi} . \quad \text { Now } \\
& e r_{E}(g) \cap g(E) \neq \phi \text { so pick } y \in e r_{E}(g) \cap g(E)
\end{aligned}
$$

such that $\{\|y-g(t)\|<\varepsilon\} \cap\{t \in E\}$ has nonzero $m_{T}$ measure. Thus

$$
\begin{aligned}
& \|x-f(t)\|<\varepsilon / 2, \text { for some } t \in E . \\
& \|f(t)-g(t)\|<a \\
& \|y-g(t)\|<\varepsilon / 2 .
\end{aligned}
$$

Thus $\|x-y\|<a+\varepsilon$.

Let $\left\{f_{n}\right\}$ be a sequence of functions from $K$ into $X$. We say that $\operatorname{er}\left(f_{n}\right)$ are bounded away from 0 over the sets $\left\{\left\|f_{n}\right\| \geqq a\right\}$ if for every $\varepsilon_{2}>0$ there exists $\varepsilon_{1}>0$ and a partition $\left\{A_{i}\right\}$ such that for all $L>0$

$$
\begin{aligned}
& \left|\sum_{i}^{\prime} m_{T}\left[A_{i} \cap\left\{\left\|f_{n}\right\|>a\right\}\right]\left(y_{i, n}\right)\right|>\varepsilon_{1} \text { imply } \\
& \quad s v\left[m_{L}, A \cap\left\{\left\|f_{n}\right\|>a\right\}\right]<\varepsilon_{2} \text {, where } A=\bigcup_{i} A_{i} .
\end{aligned}
$$

Here $\Sigma^{\prime}$ denotes the sum over $y_{\imath, n} \in X$ satisfying

$$
\left\|y_{i, n}\right\|<L \text { and } y_{i, n} \in e r_{A_{i}}\left(f_{n}\right) \text {; }
$$

Note. If $X$ denotes the scalar field the above condition will be true if $0<\delta<y_{\imath, n}<L$. That is, if the $y_{\imath, n}$ are bounded away from 0 .

Finally we say that $\operatorname{er}(h)$ transfers uniform integrability if 
for every $\varepsilon_{2}>0$ there exists $\varepsilon_{1}>0$ and constants $K_{0}>0$ and $u>0$ such that if $\left|\sum m_{T}\left(B_{i}\right) x_{i}\right|<\varepsilon_{1}$ where $\left\|x_{i}\right\| \geqq K_{0}$ and $\left\{B_{i}\right\}$ is a partition. Then $\left|\sum m_{T}\left(B_{i}\right) y_{i}\right|<\varepsilon_{2}$ provided $\left\|x_{i}-y_{i}\right\|<u$ and $y_{i} \in e r_{B_{i}}(h)$.

Note. The above condition may be rewritten

$$
\left|\int_{\|s\| \geqq K_{0}} s d m_{T}\right|<\varepsilon, \text { where } s=\sum \chi_{B_{i}} x_{i}
$$

implies $\left|\int_{|| s^{\prime}-s||<u} s^{\prime} d m_{T}\right|<\varepsilon_{2}$ where $s^{\prime}=\sum \chi_{B_{i}} y_{i}$ and $y_{i} \in e r_{B_{i}}(h)$ (in particular $\left\|y_{i}\right\| \geqq K_{0}-u$ ). Roughly speaking uniform integrability is transferred to $s^{\prime}$.

THEOREM 5. Let $\left\{f_{n}\right\}$ be a sequence of $m_{T}$ integrable functions we assume

(1) $f_{n}$ is an essential range function.

(2) $\operatorname{er}\left(f_{n}\right)$ are bounded away from 0 on sets of the form $\left\{\left\|f_{n}\right\|>a\right\}$ (for all $\left.a>0\right)$.

(3) Each er $\left(f_{n}\right)$ transfers uniform integrability.

If $\left\{\int_{A} f_{n} d m_{T}\right\}$ converges to 0 uniformly for $A \in \mathscr{B}$, then $\left\{f_{n}\right\}$ converges in measure to 0.

Proof. Let $\varepsilon>0$. Then $\left|\int_{A} f_{n} d m_{T}\right|<\varepsilon$ for all $A \in \mathscr{B}$ and for $n$ large enough. Let $\left\{s_{n, k}\right\}$ be a sequence of simple functions uniformly integrable with $\left\{s_{n, k}\right\}$ converging in measure to $f_{n}$ as $K$ goes to infinity. By (1), for $n$ fixed, we can pick a constant $M$ such that

$$
\left|\int_{\left\{\| s_{n, k} \mid>M-1\right\} \cap A} s_{n, k} d m_{T}\right|<\varepsilon \text { for all } k \text { and } A \in \mathscr{B} \text {. }
$$

Now choose $\delta^{\prime}$ so that $0<\delta^{\prime}<1 / 2$ and $\delta^{\prime}<u / 2$ and $s v_{M, 2 o^{\prime}}\left[m_{T}, K\right]<\varepsilon$. Let

$$
A_{n}^{k}\left(\delta^{\prime}\right)=\left\{t \mid\left\|s_{n, k}(t)-f_{n}(t)\right\| \geqq \delta^{\prime}\right\}
$$

Since $\left\{s_{n, k}\right\}$ converges in measure,

$$
s v_{M} m_{T}\left[A_{n}^{k}\left(\delta^{\prime}\right)\right] \text { converges to } 0 \text { as } k \rightarrow \infty .
$$

Choose $k$ large enough so that

$$
s v_{M} m_{T}\left[A_{n}^{k^{\prime}}\left(\delta^{\prime}\right) \cap E_{i, n, k}\right] \neq 0 \text { where }
$$

$A_{n}^{k^{\prime}}\left(\delta^{\prime}\right)$ denotes the complement of $A_{n}^{k}\left(\delta^{\prime}\right)$ and where $s_{n, k}=\sum_{i} E_{i, n, k} x_{i, n, k}$. Condition (1) implies $\left|\Sigma^{\prime} m_{T}\left(E_{i, n, k}\right) x_{i, n, k}\right|<\varepsilon$ where $\Sigma^{\prime}$ is the sum over $\left\|x_{i, n, k}\right\|>M-1$. Since $s_{n, k}$ is a simple function $x_{i, n, k} \in$ $e r_{s_{n, k}}\left[E_{i, n, k} \cap A_{n}^{k^{\prime}}\left(\delta^{\prime}\right)\right] . \quad$ By the previous lemma choose $y_{i, n, k} \in$ 


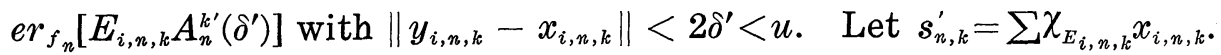
Then by condition (3)

$$
\begin{aligned}
& \left|\int s_{n, k} d m_{T}-\int s_{n, k}^{\prime} d m_{T}\right| \leqq\left|\int_{\left\{|| s_{n, k}|| \geqq M\right\}} s_{n, k} d m_{T}\right|+\left|\int_{\left\{\|\left|s_{n, k}^{\prime}\right| \mid \geqq M\right\}} s_{n, k}^{\prime} d m_{T}\right| \\
& +\left|\int_{\left.\|\| s_{n, k} \|<M\right\}} s_{n, k} d m_{T}-\int_{\left.\left\{\| s_{n, k}^{\prime} \mid<M\right\}\right\}} s_{n, k}^{\prime} d m_{T}\right| \\
& \leqq 2 \varepsilon+s v_{M, 20^{\circ}}\left[m_{T}, K\right] \text {. }
\end{aligned}
$$

Thus the right side is less than $3 \varepsilon$. Now choose $n$ large enough so that

$$
\mid \int_{\left.\left\{\left\|f_{n}\right\|>\bar{o}^{\prime}\right\} \cap\|\| s_{n, k}^{\prime} \| \leqq L\right\}} f_{n} d m_{T}<\varepsilon \text { for all } L ;
$$

(This can be done since $\left\{\int_{A} f_{n} d m_{T}\right\}$ converges to 0 uniformly for $A \in \mathscr{B}$.$) Let A=\left\{\left\|f_{n}\right\|>\delta^{\prime}\right\} \cap\left\{\left\|s_{n, k}^{\prime}\right\| \leqq L\right\}$, then shrinking $\delta^{\prime}$ if necessary

$$
\begin{aligned}
&\left|\int_{A} s_{n, k}^{\prime} d m_{T}\right| \leqq\left|\int_{A} f_{n} d m_{T}\right|+\left|\int_{A} f_{n} d m_{T}-\int_{A} s_{n, k} d m_{T}\right| \\
&+\left|\int_{A} s_{n, k} d m_{T}-\int_{A} s_{n, k}^{\prime} d m_{T}\right| \\
& \leqq 5 \varepsilon
\end{aligned}
$$

(The second term on the right is less than $\varepsilon$ since by Proposition 3 $\left\{\int_{A} s_{n, k} d m_{T}\right\}$ converges to $\int_{A} f_{n} d m_{T}$ uniformly as $A \in \mathscr{B}$.) The rest of the proof follows from condition (2).

ACKNOWLEDGMENT. We wish to thank the referee for bringing reference [14] to our attention.

\section{REFERENCES}

1. R. Alo and A. de Korvin, Representation of Hammerstein oparators by Nemytskii measures, J. Math. Analysis Appl., 152 (1975), 490-513.

2. R. Alo, A. de Korvin and Vo Van Tho, Integration theory for Hammerstein operators, J. Math. Anal. Appl., 61 (1977), 72-96.

3. J. Batt, Nonlinear integral operators on $C(S, E)$, Studia Math., 48 (1973), 145-177.

4. C. W. Burrill, Measure, Integration and Probability, McGraw-Hill Book Co., New York, 1972.

5. B. D. Coleman and V. J. Mizel, Norms and semi-groups in the theory of fading memory, Arch. Rat. Mech. Anal., 23 (1966), 87-123.

6. N. Dinculeanu, Vector Measures, Pergamon Press, Berlin, 1967.

7. N. Dunford and J. T. Schwartz, Linear Operators I: General Theory, Pure and Appl. Math. VII, Interscience, New York, 1958.

8. N. A. Friedman and A. E. Tong, On additive operators, Canad. J. Math., 23 (1971), 468-480. 
9. I. M. Gelfand and N. Ya. Vilenkin, Generalized functions, Vol. 4: Appl. Of Harmmonic Analysis, 273-278, New York, 1964,

10. R. K. Goodrich, A Riesz representing theorem in the settung of locally convexation spaces, Trans. Amer. Math. Soc., 131 (1968), 246-258.

11. I. Kluvanek, The Extension And Closure Of Vector Measures, Vector And OperatorValued Measures And Applications, Academic Press, New York, 1973, 168-183.

12. V. J. Mizel, Characterization of non-linear transformations possessing kemels, Canad. J. Math., XXII (1970), 449-471.

13. M. A. Rieffel, The Radon-Nikodym theorem for the Bochner integral, Trans. Amer. Math. Soc., 131 (1968), 466-487.

14. W. V. Smith, Convergence in measure of integrals, (to appear).

15. W. V. Smith and D H. Tucker, Weak integral convergence theorems and countable additivity, (to appear).

16. D. H. Tucker and S. G. Wayment, Absolute continuity and the Radon-Nikodym theorem, J. fur die Reine und Angew. Math., 244 (1970), 1-9.

Received October 24, 1979 and in revised form April 12, 1980.

Indiana State University

Terre Haute, IN 47809 



\section{PACIFIC JOURNAL OF MATHEMATICS}

\section{EDITORS}

DONALD BABBITT (Managing Editor)

University of California

Los Angeles, CA 90024

HUGo RossI

University of Utah

Salt Lake City, UT 84112

C. C. MOORE and ANDREW OGG

University of California

Berkeley, CA 94720
J. DUGUNDJI

Department of Mathematics

University of Southern California

Los Angeles, CA 90007

R. FinN and J. Milgram

Stanford University

Stanford, CA 94305

\section{ASSOCIATE EDITORS}
R. ARENS
E. F. BECKENBACH
B. H. NeumanN
F. WOLF
K. Yoshida

\section{SUPPORTING INSTITUTIONS}

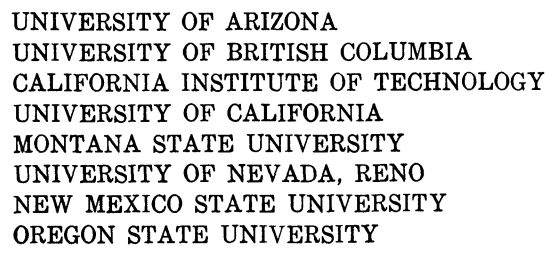

UNIVERSITY OF ARIZONA

UNIVERSITY OF BRITISH COLUMBIA CALIFORNIA INSTITUTE OF TECHNOLOGY

UNIVERSITY OF CALIFORNIA

MONTANA STATE UNIVERSITY

UNIVERSITY OF NEVADA, RENO

NEW MEXICO STATE UNIVERSITY

OREGON STATE UNIVERSITY

\author{
UNIVERSITY OF OREGON \\ UNIVERSITY OF SOUTHERN CALIFORNIA \\ STANFORD UNIVERSITY \\ UNIVERSITY OF HAWAII \\ UNIVERSITY OF TOKYO \\ UNIVERSITY OF UTAH \\ WASHINGTON STATE UNIVERSITY \\ UNIVERSITY OF WASHINGTON
}

The Supporting Institutions listed above contribute to the cost of publication of this Journal, but they are not owners or publishers and have no responsibility for its content or policies.

Mathematical papers intended for publication in the Pacific Journal of Mathematics should be in typed form or offset-reproduced, (not dittoed), double spaced with large margins. Please do not use built up fractions in the text of the manuscript. However, you may use them in the displayed equations. Underline Greek letters in red, German in green, and script in blue. The first paragraph or two must be capable of being used separately as a synopsis of the entire paper. Please propose a heading for the odd numbered pages of less than 35 characters. Manuscripts, in triplicate, may be sent to any one of the editors. Please classify according to the scheme of Math. Reviews, Index to Vol. 39. Supply name and address of author to whom proofs should be sent. All other communications should be addressed to the managing editor, or Elaine Barth, University of California, Los Angeles, California, 90024.

50 reprints to each author are provided free for each article, only if page charges have been substantially paid. Additional copies may be obtained at cost in multiples of 50 .

The Pacific Journal of Mathematics is issued monthly as of January 1966. Regular subscription rate: $\$ 102.00$ a year (6 Vols., 12 issues). Special rate: $\$ 51.00$ a year to individual members of supporting institutions.

Subscriptions, orders for numbers issued in the last three calendar years, and changes of address shoud be sent to Pacific Journal of Mathematics, P.O. Box 969, Carmel Valley, CA 93924, U.S.A Old back numbers obtainable from Kraus Periodicals Co., Route 100, Millwood, NY 10546.

\section{PUBLISHED BY PACIFIC JOURNAL OF MATHEMATICS, A NON-PROFIT CORPORATION}

Printed at Kokusai Bunken Insatsusha (International Academic Printing Co., Ltd.). 8-8, 3-chome, Takadanobaba, Shinjuku-ku, Tokyo 160, Japan. 


\section{Pacific Journal of Mathematics}

Vol. 92, No. $2 \quad$ February, 1981

Bruce Allem Anderson and Philip A. Leonard, Sequencings and Howell designs

Kevin T. Andrews, Representation of compact and weakly compact

operators on the space of Bochner integrable functions . . . . . . . . 257

James Glenn Brookshear, On the structure of hyper-real $z$-ultrafilters . . . . . 269

Frank John Forelli, Jr., A necessary condition on the extreme points of a class of holomorphic functions. II ...................... 277

Richard J. Friedlander, Basil Gordon and Peter Tannenbaum, Partitions of groups and complete mappings ......................... 283

Emden Robert Gansner, Matrix correspondences of plane partitions ......295

David Andrew Gay and William Yslas Vélez, The torsion group of a radical extension ..........................................

André (Piotrowsky) De Korvin and C. E. Roberts, Convergence theorems for some scalar valued integrals when the measure is Nemytskii ...... 329

Takaŝi Kusano and Manabu Naito, Oscillation criteria for general linear ordinary differential equations $\ldots \ldots \ldots \ldots \ldots \ldots \ldots \ldots \ldots \ldots \ldots \ldots \ldots \ldots \ldots \ldots$

Vo Thanh Liem, Homotopy dimension of some orbit spaces .......... 357

Mark Mahowald, $b o$-resolutions . . . . . . . . . . . . . . . . . . . 365

Jan van Mill and Marcel Lodewijk Johanna van de Vel, Subbases, convex

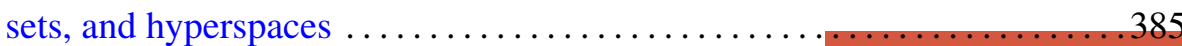

John F. Morrison, Approximations to real algebraic numbers by algebraic numbers of smaller degree $\ldots \ldots \ldots \ldots \ldots \ldots \ldots \ldots \ldots \ldots \ldots \ldots \ldots$

Caroline Series, An application of groupoid cohomology . . . . . . . . . . 415

Peter Frederick Stiller, Monodromy and invariants of elliptic surfaces . . . 433 Akihito Uchiyama, The factorization of $H^{p}$ on the space of homogeneous

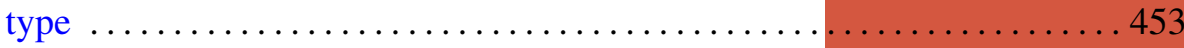

Warren James Wong, Maps on simple algebras preserving zero products.

II. Lie algebras of linear type 